\title{
Generation of Multiply Charged Peptides and Proteins by Radio Frequency Acoustic Desorption and Ionization for Mass Spectrometric Detection
}

\author{
R. Brent Dixon, Jason S. Sampson, and David C. Muddiman \\ W. M. Keck FT-ICR Mass Spectrometry Laboratory, Department of Chemistry, North Carolina State \\ University, Raleigh, North Carolina, USA
}

\begin{abstract}
The design and implementation of a radio frequency acoustic desorption ionization (RADIO) source has been demonstrated for the analysis of multiply charged peptides and proteins. One $\mu \mathrm{L}$ aliquots of melittin, BNP-32, and ubiquitin ( $\sim 1 \mu \mathrm{g}$ of analyte) were deposited onto a quartz crystal microbalance (QCM) electrode before radio frequency actuation for desorption. Continuous electrospray parallel to/above the sampling surface enabled the ionization of desorbed species. Detection by a hybrid linear ion trap Fourier transform ion cyclotron resonance mass spectrometer confirmed the intact and dissociated species observed during MS and MS/MS experiments, respectively. (J Am Soc Mass Spectrom 2009, 20, 597-600) (c) 2009 American Society for Mass Spectrometry
\end{abstract}

$\mathrm{N}$ umerous ionization techniques are being developed to address a diverse collection of complex and interesting questions by mass spectrometry (MS). Several recently developed ionization sources indirectly introduce principles of the RADIO source. Laser-induced acoustic desorption (LIAD) [1-4] demonstrates the soft desorption of analyte using a shock wave generated by a laser pulse and the potential to couple post-desorption ionization techniques such as chemical ionization at atmospheric pressure. Array of micro-machined ultrasonic electrosprays [5-7] (AMUSE), applies an rf signal to a ceramic piezoelectric transducer with a solution cavity between a micromachined silicone nozzle array for droplet-on-demand generation. The preceding techniques address RADIO's desorption mechanism. Several other techniques are mentioned below as they utilize postdesorption ionization (as in RADIO). Secondary electrospray ionization (SESI) [8-11] involves the gas-phase interaction of charged ESI droplets with neutral sample molecules for analysis by ion mobility spectrometry (IMS) or MS. Fused droplet electrospray ionization (FD-ESI) $[12,13]$ aerosolizes the sample solution via a nebulizer for interaction with a highly charged acidic methanol solution and reduces interferences from buffers and complex mixtures. Extractive electrospray ionization (EESI) [14-18] employs two nebulizing sprayers, one with ESI solvents and a second containing the analyte of interest for a liquidliquid extraction process to reduce interferences from complex mixtures.

Address reprint requests to Dr. D. C. Muddiman, W. M. Keck FT-ICR Mass Spectrometry Laboratory, Department of Chemistry, North Carolina State University, Raleigh, NC 27695, USA. E-mail: david_muddiman@ncsu.edu
Closely related to RADIO due to step-wise desorption with subsequent ionization are electrosprayassisted laser desorption ionization (ELDI) [19] and solid-state matrix assisted laser desorption electrospray ionization (ss-MALDESI) [20, 21]. ss-MALDESI utilizes a UV or IR laser for analyte desorption and ESI for post-ionization.

The desorption phenomena can be attributed in part to the shock wave desorption observed in LIAD, however for RADIO, the transfer of energy is accomplished by applying an rf waveform to a piezoelectric material (analogous to AMUSE). Postdesorption electrospray is the proposed ionization pathway as in SESI, FD-ESI, EESI, and ss-MALDESI.

\section{Experimental}

\section{Materials}

Melittin, BNP-32, ubiquitin, and formic acid were obtained from Sigma Aldrich (St. Louis, MO). HPLC grade acetonitrile and water were purchased from Burdick and Jackson (Muskegon, MI). Nitrogen (99.98\%) and LTQ helium bath gas (99.999\%) were obtained from MWSC High Purity Gases (Raleigh, NC).

\section{Methods}

Electrospray solutions consisted of acetonitrile:water ( $50: 50 \%$ by volume) with $0.1 \%$ formic acid. Melittin was diluted to $347 \mu \mathrm{M}$ in water, BNP-32 was diluted to 300 $\mu \mathrm{M}$ in water with $0.1 \%$ formic acid, and ubiquitin was diluted to $99 \mu \mathrm{M}$ in 50:50:0.1\% water:acetonitrile:formic acid. QCM substrates were spotted with $1 \mu \mathrm{L}$ of the 
respective analyte immediately before analysis. This resulted in $\sim 1 \mu \mathrm{g}$ of analyte on the surface with a minute fraction sampled by the mass spectrometer.

\section{Mass Spectrometer}

Mass spectra were acquired on a hybrid LTQ-FT-ICR mass spectrometer (Thermo Fisher, San Jose, CA) equipped with an Oxford Instruments actively shielded 7T superconducting magnet (Concord, MA) operating in positive ion, FT mode. The injection time was set to $750 \mathrm{~ms}$ (BNP-32 and ubiquitin) and $500 \mathrm{~ms}$ (melittin) for full scan experiments (AGC off). For MS/MS, the injection time was set to $1000 \mathrm{~ms}$ (AGC off); product ions generated in the ion trap were transferred to the FT-ICR cell for detection. The capillary temperature was $250{ }^{\circ} \mathrm{C}$. The standard MS capillary was replaced with a $0.5 \mathrm{~mm}$ i.d. $\times 131 \mathrm{~mm}$ stainless steel capillary that protruded 32 $\mathrm{mm}$ from the ferrule.

\section{RADIO Source}

The experiments utilized an AG 1020 "Linear" rf Amplifier/Generator (P/N AG1020; T and C Power Conversion, Inc., Rochester, NY) to actuate a quartz crystal microbalance (QCM) electrode (P/N 151689-10; International Crystal Manufacturing, Inc., Oklahoma City, $\mathrm{OK})$. The surface of the QCM electrodes were Au plated Cr. Figure 1 displays the RADIO source components. The rf amplifier is shown connected to the QCM electrode with the electrospray emitter positioned above the sample spot such that the ESI plume is directed into the mass spectrometer. QCM electrodes were mounted on a Teflon bracket attached to an XYZ stage for position adjustment. The rf amplifier was connected to the QCM electrode via a BNC cable with mini-clip jumper wires. The resonance frequency to generate

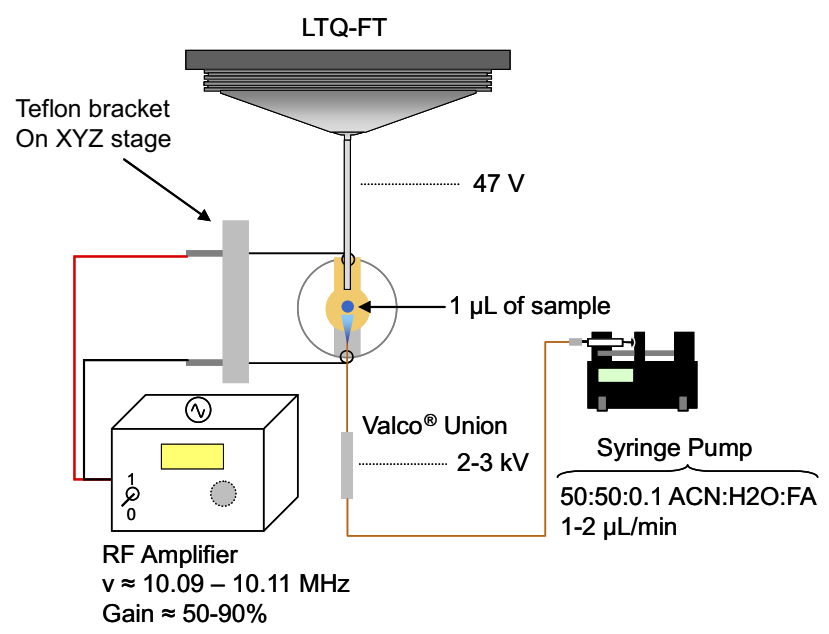

Figure 1. Schematic of the RADIO source showing the experimental configuration with the rf amplifier attached to the QCM electrode; the ESI emitter is located above the QCM electrode with flow directed at the MS inlet. progeny droplets from a $1 \mu \mathrm{L}$ sample on the QCM surface was experimentally modulated. At an applied rf of 10.09 to $10.11 \mathrm{MHz}$, and a gain of $60 \%$ to $90 \%$, mass spectrometric signal was observed. No DC bias was applied to the QCM.

Solvent was electrosprayed continuously above the electrode surface during MS analysis for droplet interaction and ionization with the electrospray plume (vide infra). The electrospray parameters were $1-2 \mu \mathrm{L} / \mathrm{min}$ through a $30 \mu \mathrm{m}$ electrospray emitter biased at 2 to 3 $\mathrm{kV}$. The mass spectrometer inlet was biased at $47 \mathrm{~V}$. The QCM electrode was cleaned between analyte depositions and subsequent acquisitions by rinsing $10 \mu \mathrm{L}$ of 50:50:0.1\% water:acetonitrile:formic acid and drying the surface with compressed air.

Control experiments were performed to ensure that signal resulted from droplet generation off of the QCM surface. The first involved turning off the rf amplifier with a sample droplet present on the surface, the second involved entirely removing the QCM electrode, and in a third, a $1 \mu \mathrm{L}$ aliquot of solvent was deposited onto the QCM surface and the rf amplifier signal was turned on. During each control experiment, a continuous stream of solvent was electrosprayed and the MS signal was monitored with no detection of analyte. Preventative cleaning of the ESI emitter and MS inlet with an acetonitrile soaked cotton swab prevented cross-contamination between samples.

\section{Results and Discussion}

The RADIO source is illustrated in Figure 1. The positioning of the QCM electrode with respect to the MS inlet and ESI emitter was facilitated by a XYZ stage. Constant spray current was observed with intense spikes of signal on a total ion chronogram when desorbed species were detected by the mass spectrometer (data not shown).

Full scan mass spectra were obtained by depositing $1 \mu \mathrm{L}$ of analyte before rf actuation of the QCM electrode. By constantly electrospraying parallel to the sample surface (above the analyte spot) during the desorption event, desorbed species interacted with the ESI droplets for ionization. This is analogous to ionization in fused droplet-electrospray ionization (FD-ESI) [12, 13] and extractive electrospray ionization (EESI) [14-18] due to neutral interaction with an ESI plume. Previous reports demonstrated that postdesorption ionization offers the advantage of withstanding high salt tolerances [12]. The interaction time of desorbed species with the ESI plume is one factor that can be attenuated by adjusting the linear distance of the ESI emitter from the MS inlet (holding the sample spot constant). For these experiments, an ESI emitter to MS inlet distance of $\sim 5 \mathrm{~mm}$ was utilized.

Figure 2 demonstrates multiple-charging, high signalto-noise, and isotopic resolution of brain natriuretic peptide-32 (BNP-32) [22] attained using RADIO. The 


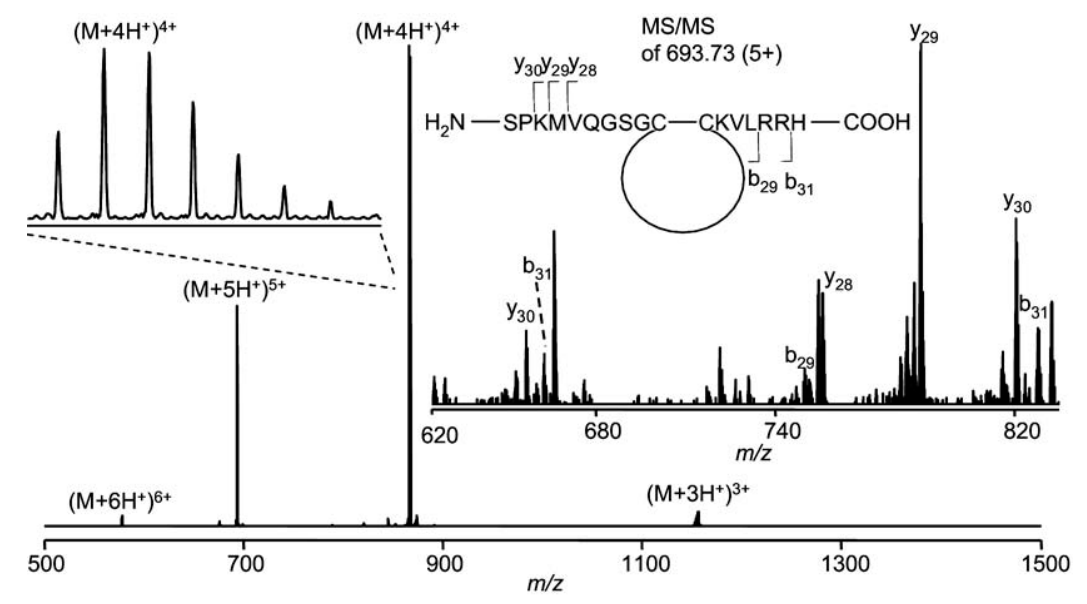

Figure 2. Intact FT-ICR detection of BNP-32 with isotopic distribution of the $4+$ charge state and inset of FT-ICR detection of CID fragmentation of $5+$ charge state.

$3+$ through $6+$ charge states are shown with a zoom-in of the isotopic distribution of the $4+$ charge state. Accurate mass measurements indicated that BNP-32 was oxidized as reported previously by Hawkridge et al. [22] This is a result of a cysteine-cysteine disulfide bond linkage, which forms a ring-like structure (see sequence depiction in Figure 2). MS/MS was carried out on the $4+$ charge state. A representative collision induced dissociation (CID) spectra is inset in Figure 2. The number of product ions observed is attributed in part to the oxidized form of BNP-32.

Full scan mass spectra were obtained from a $1 \mu \mathrm{L}$ sample of ubiquitin deposited onto the QCM electrode. A representative mass spectrum is shown in Figure 3 including the charge states of $6+$ through $12+$. MS/MS data of the $7+$ precursor ion is inset in Figure 3. Additional data were collected including full scan detection of the $3+$ and $4+$ charge states of melittin (data not shown).

\section{Conclusions}

An ambient ionization source with multiple-charging for MS and MS/MS analyses has been described. The use of a rf amplifier for analyte desorption should enable a variety of ambient ionization mass spectrometry applications. This proof-of-principle demonstration of the RADIO source should pique the interest of investigators as a method to address complex biological questions.

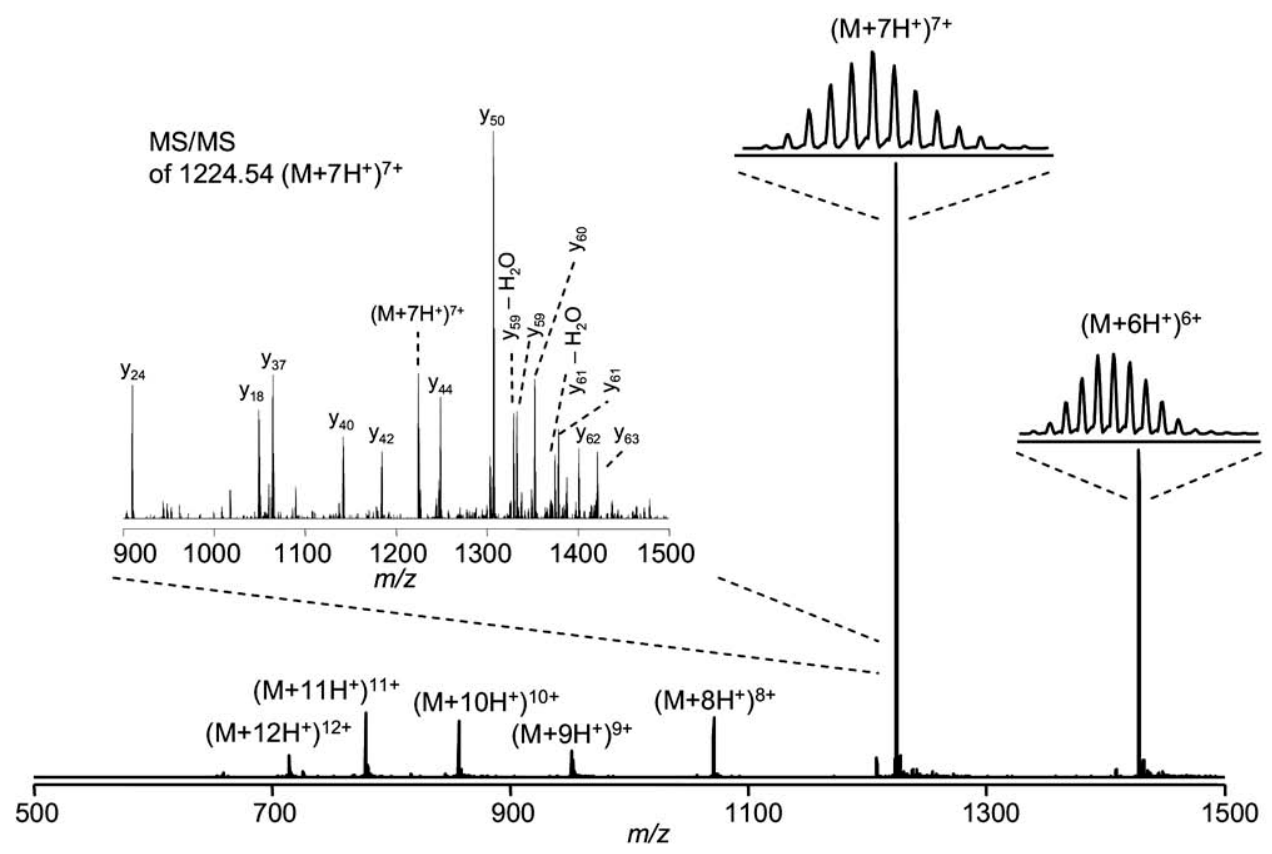

Figure 3. Intact FT-ICR detection of ubiquitin with isotopic distribution of the $6+$ and $7+$ charge states and inset of FT-ICR detection of CID fragmentation of 7+ charge state. 


\section{Acknowledgments}

The authors gratefully acknowledge financial support received from the W. M. Keck Foundation and North Carolina State University.

\section{References}

1. Lindner, B., Laser Desorption Mass Spectrometry of Nonvolatiles Under Shock Wave Conditions. Anal. Chem. 1985, 57(4), 895-899.

2. Golovlev, V. V.; Allman, S. L.; Garrett, W. R.; Chen, C. H. Laser-Induced Acoustic Desorption of Electrons and Ions. Appl. Phys. Lett. 1997, 71(6), 852-854

3. Perez, J.; Ramirez-Arizmendi, L. E.; Petzold, C. J.; Guler, L. P.; Nelson, E. D.; Kenttamaa, H. I. Laser-Induced Acoustic Desorption/Chemical Ionization in Fourier-Transform Ion Cyclotron Resonance Mass Spectrometry. Int. J. Mass Spectrom. 2000, 198(3), 173-188.

4. Duan, P.; Qian, K.; Habicht, S. C.; Pinkston, D. S.; Fu, M. K.; Kenttamaa, H. I. Analysis of Base Oil Fractions by $\mathrm{ClMn}(\mathrm{H} 2 \mathrm{O})(+)$ Chemical Ionization Combined with Laser-Induced Acoustic Desorption/Fourier Transform Ion Cyclotron Resonance Mass Spectrometry. Anal. Chem. 2008, 80(6), 1847-1853.

5. Meacham, J. M.; Ejimofor, C.: Kumar, S.; Degertekin, F. L.; Fedorov, A. G. Micromachined Ultrasonic Droplet Generator Based on a Liquid Horn Structure. Rev. Sci. Instrum. 2004, 75(5), 1347-1352.

6. Aderogba, S.; Meacham, J. M.; Degertekin, F. L.; Fedorov, A. G.; Fernandez, F. M. Nanoelectrospray Ion Generation for High-Throughput Mass Spectrometry Using a Micromachined Ultrasonic Ejector Array. Appl. Phys. Lett. 2005, 86(20), 203110-203113.

7. Hampton, C. Y.; Forbes, T. P.; Varady, M. J.; Meacham, J. M.; Fedorov, A. G.; Degertekin, F. L.; Fernandez, F. M. Analytical Performance of a Venturi-Assisted Array of Micromachined Ultrasonic Electrosprays Coupled to Ion Trap Mass Spectrometry for the Analysis of Peptides and Proteins. Anal. Chem. 2007, 79(21), 8154-8161.

8. Chen, Y. H.; Hill, H. H.; Wittmer, D. P. Analytical Merit of Electrospray Ion Mobility Spectrometry as a Chromatographic Detector. J. Microcolumn Sep. 1994, 6(5), 515-524.

9. Wu, C.; Siems, W. F.; Hill, H. H. Secondary Electrospray Ionization Ion Mobility Spectrometry/Mass Spectrometry of Illicit Drugs. Anal. Chem. 2000, 72(2), 396-403.

10. Steiner, W. E.; Clowers, B. H.; Haigh, P. E.; Hill, H. H. Secondary Ionization of Chemical Warfare Agent Simulants: Atmospheric Pressure Ion Mobility Time-of-Flight Mass Spectrometry. Anal. Chem. 2003, 75(22), 6068-6076.
11. Tam, M.; Hill, H. H. Secondary Electrospray Ionization-Ion Mobility Spectrometry for Explosive Vapor Detection. Anal. Chem. 2004, 76(10) 2741-2747.

12. Chang, D. Y.; Lee, C. C.; Shiea, J. Detecting Large Biomolecules from High-Salt Solutions by Fused-Droplet Electrospray Ionization Mass Spectrometry. Anal. Chem. 2002, 74(11), 2465-2469.

13. Shieh, I. F. Lee, C. Y.; Shiea, J. Eliminating the Interferences from TRIS Buffer and SDS in Protein Analysis by Fused-Droplet Electrospray Ionization Mass Spectrometry. J. Proteome Res. 2005, 4(2), 606-612.

14. Chen, H. W.; Venter, A.; Cooks, R. G. Extractive Electrospray Ionization for Direct Analysis of Undiluted Urine, Milk and Other Complex Mixtures without Sample Preparation. Chem. Commun. 2006, 19, 2042 2044.

15. Chen, H. W.; Sun, Y. P.; Wortmann, A.; Gu, H. W.; Zenobi, R. Differentiation of Maturity and Quality of Fruit Using Noninvasive Extractive Electrospray Ionization Quadrupole Time-of-Flight Mass Spectrometry. Anal. Chem. 2007, 79(4), 1447-1455.

16. Chen, H. W.; Wortmann, A.; Zenobi, R. Neutral Desorption Sampling Coupled to Extractive Electrospray Ionization Mass Spectrometry for Rapid Differentiation of Biosamples by Metabolomic Fingerprinting. J. Mass Spectrom. 2007, 42(9), 1123-1135.

17. Chen, H.; Yang, S.; Wortmann, A.; Zenobi, R. Neutral Desorption Sampling of Living Objects for Rapid Analysis by Extractive Electrospray Ionization Mass Spectrometry. Angew. Chem. Int. Edit. 2007, 46(40), 7591-7594.

18. Chen, H. W.; Touboul, D.; Jecklin, M. C.; Zheng, J.; Luo, M. B.; Zenobi, R. N. Manipulation of Charge States of Biopolymer Ions by Atmospheric Pressure Ion/Molecule Reactions Implemented in an Extractive Electrospray Ionization Source. Eur. J. Mass Spectrom. 2007, 13(4), 273-279.

19. Shiea, J; Huang, M. Z.; Hsu, H. J.; Lee, C. Y.; Yuan, C. H.; Beech, I. Sunner, J. Electrospray-Assisted Laser Desorption/Ionization Mass Spectrometry for Direct Ambient Analysis of Solids. Rapid Commun. Mass Spectrom. 2005, 19(24), 3701-3704.

20. Sampson, J. S.; Hawkridge, A. M.; Muddiman, D. C. Generation and Detection of Multiply-Charged Peptides and Proteins by Matrix-Assisted Laser Desorption Electrospray Ionization (MALDESI) Fourier Transform Ion Cyclotron Resonance Mass Spectrometry. J. Am. Soc. Mass Spectrom. 2006, 17(12), 1712-1716.

21. Sampson, J. S.; Hawkridge, A. M.; Muddiman, D. C. Direct Characterization of Intact Polypeptides by Matrix Assisted Laser Desorption Electrospray Ionization (MALDESI) Quadrupole Fourier Transform Ion Cyclotron Resonance Mass Spectrometry. Rapid Commun. Mass Spectrom. 2007, 21(7), 1150-1154.

22. Hawkridge, A. M.; Heublein, D. M.; Bergen, H. R.; Cataliotti, A.; Burnett, J. C.; Muddiman, D. C. Quantitative Mass Spectral Evidence for the Absence of Circulating Brain Natriuretic Peptide (BNP-32) in Severe Human Heart Failure. Proc. Natl. Acad. Sci. U.S.A. 2005, 102(48), 17442-17447. 